\title{
Generating attosecond x-ray pulses through an angular dispersion enhanced self-amplified spontaneous emission free electron laser
}

\author{
Zheng Qi, ${ }^{1,2}$ Chao Feng, ${ }^{1, *}$ Haixiao Deng, ${ }^{1}$ Bo Liu, ${ }^{1}$ and Zhentang Zhao ${ }^{1, \dagger}$ \\ ${ }^{1}$ Shanghai Institute of Applied Physics, Chinese Academy of Sciences, Shanghai 201800, China \\ ${ }^{2}$ University of Chinese Academy of Sciences, Beijing 100049, China
}

(Received 23 October 2018; published 26 December 2018)

\begin{abstract}
We propose a scheme that can generate attosecond $\mathrm{x}$-ray pulses through an angular dispersion enhanced self-amplified spontaneous emission (ESASE) free electron laser (FEL). In this scheme, an angular dispersion section is implemented into a conventional ESASE set-up, together with a few cycle laser pulse to manipulate the electron beam effectively. During the procedure, the transverse-longitudinal phase space coupling is introduced into the electron beam in addition to the energy modulation and momentum compaction processes. As a result, the peak current of the electron beam will be strengthened significantly within a narrow width of the electron bunch, which can be used to generate ultrashort FEL pulses. Comparing to a conventional ESASE scheme, the proposed scheme can reduce the required modulation laser power by at least one order of magnitude. The signal-to-noise ratio of the final FEL radiation pulses can also be improved. In addition, we discuss the possibility of using this scheme to generate two-pulse two-color x-ray FEL with tunable central wavelengths and time delays for a conceivable $\mathrm{x}$-ray pump/x-ray probe experiment.
\end{abstract}

DOI: 10.1103/PhysRevAccelBeams.21.120703

\section{INTRODUCTION}

The pump-probe technique is widely used to investigate the fast dynamic phenomena within chemical, physical, and biological reactions, in which the pump laser initiates the excitation of the sample and the probe laser with a certain time delay characterizes the changes [1-4]. The experimental methodology of this technique keeps on developing. The resolution of the time-resolved pump-probe experiments depends on the duration of the pump and probe lasers. The ultrashort laser pulses down to attosecond level make it possible to study the ultrafast electronic dynamics. And the advanced short-wavelength x-ray lasers open up a new world of the atomic inner-shell electronic transitions. Given these circumstances, the generation of attosecond $\mathrm{x}$-ray pump and $\mathrm{x}$-ray probe laser pulses is of great interests in the scientific community. Moreover, the central wavelengths of these two lasers and the time delays between them are preferred to be adjustable. The conventional laser system can hardly fulfill the task in terms of all these requirements. However, the free electron lasers (FELs)

\footnotetext{
*fengchao@sinap.ac.cn

'zhaozhentang@sinap.ac.cn
}

Published by the American Physical Society under the terms of the Creative Commons Attribution 4.0 International license. Further distribution of this work must maintain attribution to the author(s) and the published article's title, journal citation, and DOI.
[5-7] might have the ability to deliver such controllable attosecond two-color x-ray laser pulses.

The free electron lasers (FELs) have been served as a great tool in the state-of-the-art researches in many scientific frontiers [8-10]. FELs can provide ultrahigh intensity ultrashort pulse duration radiation with tuneable central wavelength ranging from terahertz to $\mathrm{x}$-ray. There are several $\mathrm{x}$-ray FEL facilities under operation around the world. And their success has encouraged the world to develop and construct more advanced FEL facilities. At present, these facilities are mainly based on the self-amplified spontaneous emission (SASE) scheme [11,12], in which the initial signal originating from the electron beam shot noise is amplified the way up to saturation along the undulator. The typical pulse configuration of a SASE-FEL is about tens of GW peak power and tens to one hundred femtoseconds pulse duration. The pulse duration is generally determined by the lasing part of the electron bunch. In order to deliver ultrashort FEL pulses, the lasing part within the electron bunch should be as short as possible.

Several methods or techniques have been proposed to shorten the FEL pulse duration on the basis of the SASEFEL [13-19]. The basic idea is to make sure only a small part of the electron beam will lase while other parts remain suppressed. One can either use a slotted foil [17] to directly select that part, or employ an external few-cycle laser pulse to help form that part [14]. The latter is usually termed as the enhanced SASE (ESASE) scheme. LCLS has been working on a project to develop the ESASE technique 
recently. The peak power of the FEL radiation can be further improved by combining the ESASE technique with the mode-lock concept (for example in [20-22]), from which the terawatt-attosecond FEL pulses might be achievable. However, in order to generate attosecond radiation pulses, the external few-cycle laser power used in a conventional ESASE scheme needs to be as high as tens of GW, which is quite difficult to be supplied and becomes rather impractical when it comes to high rep-rate FEL facilities.

Hence in this paper, we propose a modified version of the ESASE scheme to reduce the required power of the external seed laser, and the signal-to-noise ratio of the final FEL pulses can also be improved. The proposed scheme borrows the idea from [23] of introducing angular dispersion into the electron beam in addition to the energy modulation and momentum compaction processes, so that we could manipulate the longitudinal electron beam distribution more effectively. We also discuss the possibility of using this scheme to generate two-pulse two-color x-ray FEL with tunable central wavelengths and time delays for a conceivable $\mathrm{x}$-ray pump/x-ray probe experiment.

\section{PRINCIPLE}

The layout of our proposed scheme is shown in Fig. 1. The whole beamline consists of an angular dispersion (AD) section, a modulator section (M), a dogleg (D) and a radiator section $(\mathrm{R})$. The AD section employed here is used to introduce a transverse angular dispersion into the electron beam, through which the horizontal beam divergence will form a linear correlation with respect to the beam energy. Basically the AD section is composed of two quadrupoles laying respectively in front and in the middle of a laser-injection chicane. Then the electron beam will interact with a few cycle laser pulse in a single-period modulator (M) section to get an energy modulation. The laser pulse is injected into the $M$ section through the interstice of the AD section. After these processes, the electron beam will have a transverse angular dispersion throughout the whole bunch and a few cycle energy modulation within only a small part of the electron beam. Following the $\mathrm{M}$ section, a dogleg (D) here will provide a longitudinal dispersion to convert the energy modulation into density modulation, and if the transverse dispersion of the dogleg (D) together with the transverse angular dispersion of the $\mathrm{AD}$ section are chosen properly, the initial beam energy spread will by then no longer have impacts on the density modulation results, and a much more strengthened current spike will be formed within the electron beam. Finally the electron beam will go through the radiator $(\mathrm{R})$ section for the generation of ultrashort FEL pulses.

We will give a theoretical analysis below to explain the principle of our proposed scheme. To simplify the derivation processes and for the clearness of the theory, we assume the initial longitudinal and transverse phase space distribution to be Gaussian profile, and we only take care of the $X^{\prime}$ dimension and the $P$ dimension cause they are what really matters in this scheme. First of all, we need to define some variables. The dimensionless energy deviation of a particle is defined as $p=\left(E-E_{0}\right) / \sigma_{E}$, where $E_{0}$ is the central energy of the electron beam and $\sigma_{E}$ is the rms energy spread. The dimensionless horizontal beam divergence reads $\chi^{\prime}=\left(x^{\prime}-x_{0}^{\prime}\right) / \sigma_{x^{\prime}}$, in which $x_{0}^{\prime}$ is the average horizontal beam divergence and $\sigma_{x^{\prime}}$ is the rms beam divergence. Therefore the initial longitudinal and transverse distributions of the electron beam at the entrance of the beamline can be expressed as $f_{0}(p)=N_{0} \exp \left(-p^{2} / 2\right) / \sqrt{2 \pi}$ and $g_{0}\left(\chi^{\prime}\right)=N_{0} \exp \left(-\chi^{\prime 2} / 2\right) / \sqrt{2 \pi}$ respectively, where $N_{0}$ is the electron number per unit length of the beam.

First the electron beam will go through the $\mathrm{AD}$ section. For two quadrupoles with identical strength $Q$ and a chicane with bending angle $b$, drift length between B1-B2 and B3-B4 being $L_{1}$, drift length between B2-B3 being $2 L_{2}$, the length between the first quadrupole and $\mathrm{B} 1$ being $L_{1}$, the second quadrupole being in the middle of the chicane, the simplified four dimensional transport matrix of the $\mathrm{AD}$ section regarding $\left(\chi, \chi^{\prime}, z, p\right)$ in $X^{\prime}-P$ dimension reads now

$$
R_{A D}=\left[\begin{array}{cccc}
-1 & L_{1} & 0 & b_{1}\left(L_{1}+L_{2}\right) \\
0 & -1 & 0 & b_{1} \\
b_{1} & -2 b L_{1} & 1 & 0 \\
0 & 0 & 0 & 1
\end{array}\right]
$$

in which $b_{1}=2 b L_{1} /\left(2 L_{1}+L_{2}\right)$ represents the angular dispersion of the $\mathrm{AD}$ section. There exits a matching condition that $Q=-2 /\left(2 L_{1}+L_{2}\right)$. After passing through the $\mathrm{AD}$ section, $\chi^{\prime}$ is changed to

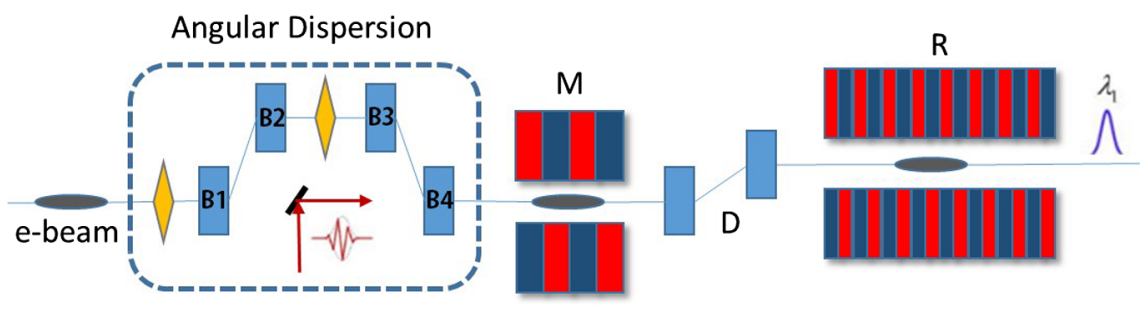

FIG. 1. Layout of our proposed scheme. 


$$
\chi_{1}^{\prime}=-\chi^{\prime}+b_{1} p \cdot \frac{\sigma_{E}}{E_{0}}
$$

It means that there exits a linear correlation between the horizontal beam divergence and the beam energy, and the transverse-longitudinal phase space coupling of the electron beam has been developed. And in the modulator (M) section, the energy modulation induced by the laser is

$$
p_{1}=p+A \sin \left(k_{0} z_{1}\right) \exp \left(-\frac{1}{2} z_{1}^{2} / \sigma_{z_{0}}^{2}\right)
$$

where $k_{0}=2 \pi / \lambda_{0}, \lambda_{0}$ and $\sigma_{z_{0}}$ are respectively the wavelength and the rms pulse length of the laser, $A=\Delta E / \sigma_{E}$ is the amplitude of the modulation strength, $z_{1}$ is the longitudinal coordinate of the electron after passing through the $\mathrm{AD}$ section. We ignore the momentum compaction factor introduced by the modulator here because it is relatively too small. In the dogleg (D), energy modulation will be converted into density modulation. Additionally, the transverse dispersion of the dogleg working together with the angular dispersion of the electron beam will also influence the density modulation processes. Hence, the longitudinal coordinate $z_{2}$ now turns into

$$
z_{2}=z_{1}+\xi_{d} p_{1} \cdot \frac{\sigma_{E}}{E_{0}}+\eta \chi_{1}^{\prime}
$$

where $\xi_{d}$ and $\eta$ are respectively the longitudinal and transverse dispersion of the dogleg. The electron beam distribution in the longitudinal phase space and transverse dimension now reads

$$
\begin{aligned}
& f_{1}\left(\zeta, \chi_{1}^{\prime}, p_{1}\right)=\frac{N_{0}}{\sqrt{2 \pi}} \exp \left\{-\frac{1}{2}\left[p_{1}-A \sin \left(\zeta-k_{0} \eta \chi_{1}^{\prime}-B p_{1}\right) \exp \left[-\frac{1}{2 \sigma_{z_{0}}^{2}}\left(\zeta-k_{0} \eta \chi_{1}^{\prime}-B p_{1}\right)^{2}\right]\right]^{2}\right\} \\
& g_{1}\left(\zeta, p_{1}, \chi_{1}^{\prime}\right)=\frac{N_{0}}{\sqrt{2 \pi}} \exp \left\{-\frac{1}{2}\left[\chi_{1}^{\prime}+b \cdot \frac{\sigma_{E}}{E_{0}} \cdot\left\{p_{1}-A \sin \left(\zeta-k_{0} \eta \chi_{1}^{\prime}-B p_{1}\right) \exp \left[-\frac{1}{2 \sigma_{z_{0}}^{2}}\left(\zeta-k_{0} \eta \chi_{1}^{\prime}-B p_{1}\right)^{2}\right]\right\}\right]^{2}\right\}
\end{aligned}
$$

where $\zeta=k_{0} z$ is the new phase of the electron, $B=k_{0} \xi_{d} \sigma_{E} / E_{0}$. Combining these two equations in Eq. (5), we can get a two-dimensional distribution of the electron beam

$$
h\left(\zeta, p_{1}, \chi_{1}^{\prime}\right)=f_{0}\left(\zeta, \chi_{1}^{\prime}, p_{1}\right) g_{0}\left(\zeta, p_{1}, \chi_{1}^{\prime}\right)
$$

Integrating Eq. (6) over $p_{1}$ and $\chi_{1}^{\prime}$ will give us the current distribution of the electron beam

$$
\begin{aligned}
\frac{N(\zeta)}{N_{0}}= & 1+2 \sum_{n=1}^{\infty} J_{n}\left[n A B \cdot \exp \left(-\frac{\zeta^{2}}{2 \sigma_{z_{0}}^{2}}\right)\right] \\
& \times \exp \left[-\frac{1}{2} n^{2}\left(B+b_{1} D\right)^{2}\right] \\
& \times \exp \left[-\frac{1}{2}\left(n k_{0} \eta \sigma_{x^{\prime}}\right)^{2}\right] \cos (n \zeta)
\end{aligned}
$$

where $D=k_{0} \eta \sigma_{E} / E_{0}$.

Apparently there exits an optimized condition for the bunching factor when $B+b_{1} D=0$, i.e.,

$$
b_{1}=-\frac{\xi_{d}}{\eta}
$$

and then the current distribution will be determined by the modulation strength $A$, the transverse dispersion of the $\operatorname{dogleg} \eta$ and the initial horizontal beam divergence $\sigma_{x^{\prime}}$. The current distribution now reads

$$
\begin{aligned}
\frac{N(\zeta)}{N_{0}}= & 1+2 \sum_{n=1}^{\infty} J_{n}\left[n A B \cdot \exp \left(-\frac{\zeta^{2}}{2 \sigma_{z_{0}}^{2}}\right)\right] \\
& \times \exp \left[-\frac{1}{2}\left(n k_{0} \eta \sigma_{x^{\prime}}\right)^{2}\right] \cos (n \zeta)
\end{aligned}
$$

One can find that the smaller the product $\eta \sigma_{x^{\prime}}$ is, the higher the peak current will be. And the current width of the optimized current peak is approximately

$$
\tau \approx \pi \eta \sigma_{x^{\prime}}
$$

where the $\eta \sigma_{x^{\prime}}$ can be very small and thus will result in a very short current spike. Since the duration of the current spike has no relation with the initial beam energy spread, very high peak current can be generated with very small laser induced energy spread. It is worthwhile to point out that Eq. (10) also sets a limitation for the maximal slippage length, within which the radiation can keep interacting with the electron beam and get amplified.

To clearly illustrate the physical mechanism of our proposed scheme, we give schematic diagrams of the electron beam longitudinal phase space evolution in Fig. 2, showing respectively the phase space right after the AD section, the modulator section and the dogleg. In the $\mathrm{AD}$ section, the electron beam will get a linear correlation between horizontal divergence and electron energy, yet the longitudinal phase space remains unchanged, as shown in Fig. 2(a). After interacting with a few cycle laser pulse in the modulator section, Fig. 2(b) demonstrates the induced energy modulation profile of the electron beam, i.e., the 


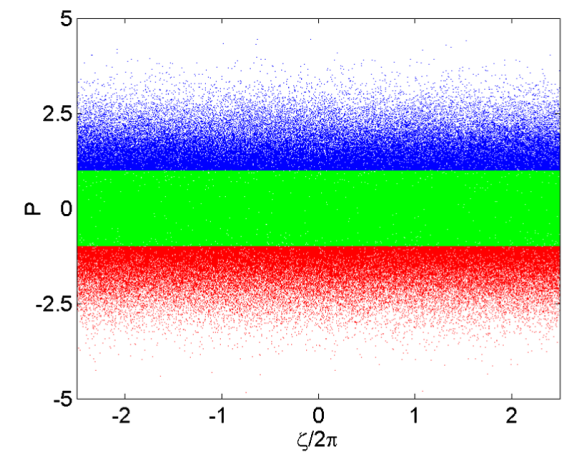

(a)

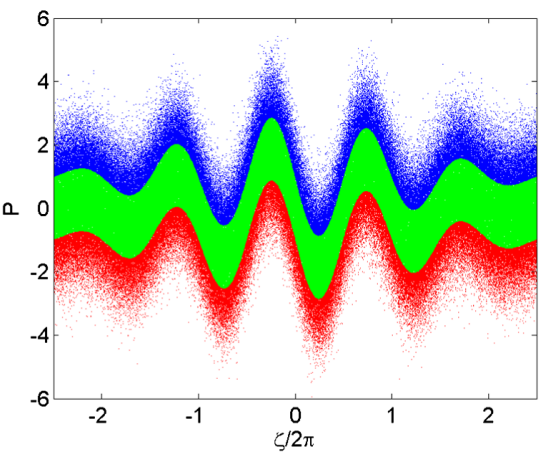

(b)

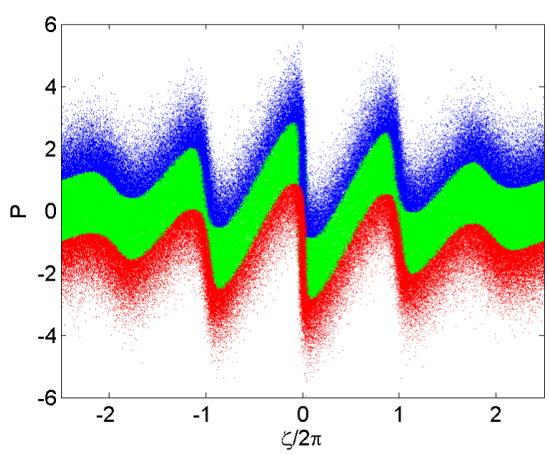

(c)

FIG. 2. Longitudinal phase space of the electron beam right after (a) the AD section, (b) the modulator section, and (c) the dogleg.

energy modulation strength will vary with respect to different longitudinal positions. And finally we can see from Fig. 2(c) the density modulation has been produced, and the initial energy spread of the electron beam will not influence the ultimate density modulation results, which will further strengthen the peak current enhancement in the ESASE technique.

Our proposed method has several advantages over the conventional ESASE technique. As we have known, in the limitation of that the practical laser pulse used in a conventional ESASE scheme could not be as short and powerful as we want, the present ESASE technique can not work so well as we expect. However in the proposed scheme, by introducing the angular dispersion, we get another dimension that could help manipulate the electron beam. And through optimization, the needed laser power can be reduced by at least one order of magnitude. Furthermore, the reduction of the seed laser power enables the possibility of using a relatively longer wavelength seed laser with fewer optical cycles in our scheme, which will help to improve the signal-to-noise ratio of the final FEL pulses significantly.

\section{MAIN PARAMETERS}

The descriptions above give us a hint about the principle and the physical mechanism of the proposed scheme. Following we will show the possible performance of the

TABLE I. Main parameters of SHINE.

\begin{tabular}{lcc}
\hline \hline Parameters & Value & Unit \\
\hline Beam energy & 8 & $\mathrm{GeV}$ \\
Slice energy spread & 0.01 & $\%$ \\
Normalized emittance & 0.45 & $\mu \mathrm{m} \cdot \mathrm{rad}$ \\
Peak current & 3 & $\mathrm{kA}$ \\
Modulator period & 60 & $\mathrm{~cm}$ \\
Period number of modulator & 1 & $\cdots$ \\
Radiator period & 26 & $\mathrm{~mm}$ \\
Period number of radiator & 100 & $\cdots$ \\
\hline \hline
\end{tabular}

scheme in a practical FEL facility. That will include the parameter optimization process and the FEL radiation simulation process. The basic parameters of the electron beam and the beamline are from the Shanghai High Repetition Rate XFEL and Extreme Light Facility (SHINE) [24], as shown in Table I. The laser pulse we employ in the modulator section is about $\sigma_{z_{0}}=4.5 \mathrm{fs}$ with a central wavelength of $\lambda_{0}=1200 \mathrm{~nm}$. The FWHM of the laser pulse intensity corresponds to about 1.9 laser cycles. We focus the laser in the middle of the modulator. And the carrier envelop phase of the laser pulse is adjusted to be sinelike and remains stabilized, so that the electron beam will get a proper energy modulation.

To lower the required laser power in a practical high rep-rate FEL facility and to show the superiority of our proposed scheme, the peak power of the laser pulse we used in the simulation is about $2.6 \mathrm{GW}$, which is an order of magnitude lower than that being used in previous ESASE studies (for example in [15]). The impact of the laser beam waist diameter has on the energy modulation strength $A$ is shown in Fig. 3, from which one can find that the maximal

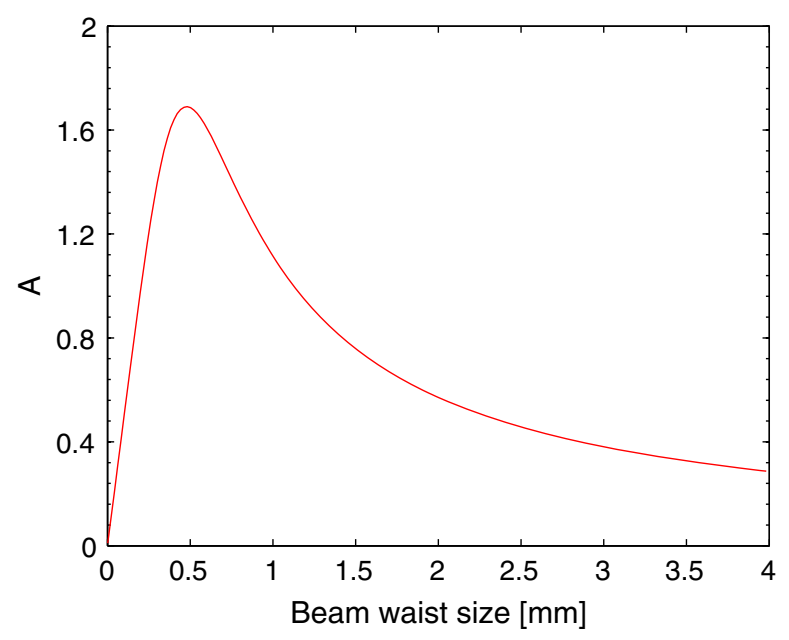

FIG. 3. Energy modulation strength with respect to different laser beam sizes. 


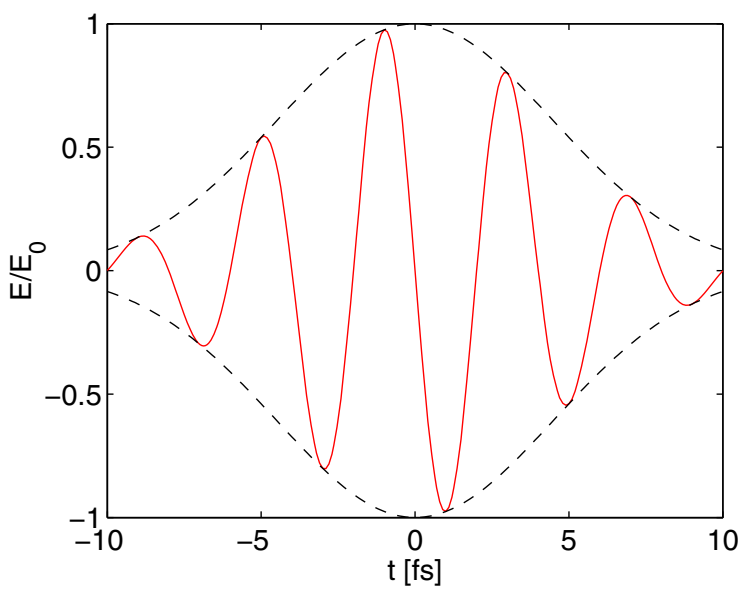

(a)

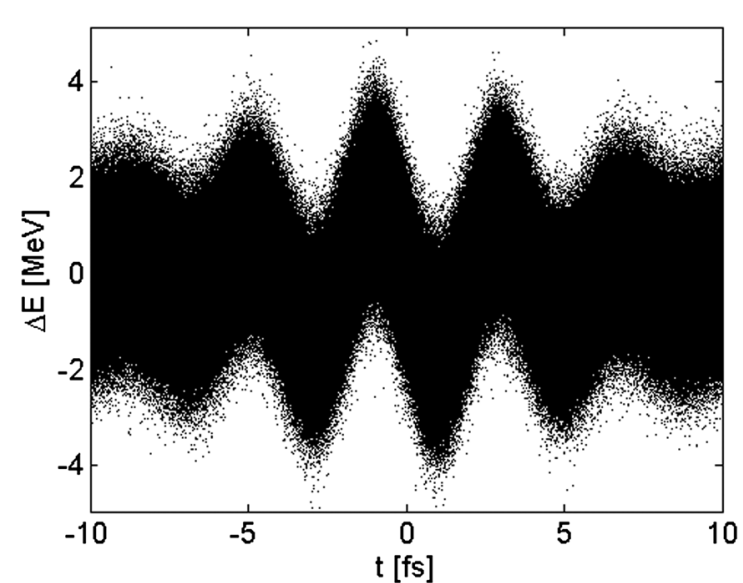

(b)

FIG. 4. (a) Normalized electric field of the laser pulse. (b) Energy change of the electron beam induced by the laser pulse.

energy modulation amplitude $A$ is about 1.8 times of the initial energy spread when the laser beam size is $0.5 \mathrm{~mm}$. And the corresponding dispersion $\xi_{d}$ is needed to be about $1.1 \mathrm{~mm}$. Figure 4 shows the electric field of the laser pulse normalized to the peak value and the energy change of the electron beam induced by the laser. The heads of the laser pulse and the electron bunch are both to the right of the figures. The zero crossing of the energy modulation is at the maximum of the laser pulse carrier envelope. And the energy chirp around this point is negative.

Then the current distribution in Eq. (9) now determines by the product of $\eta \sigma_{x^{\prime}}$. For different current width $\tau$ in Eq. (10) which can represent $\eta \sigma_{x^{\prime}}$, we depict the current distribution in Fig. 5, in which the initial current is about $3 \mathrm{kA}$. We can see with the decrease of $\tau$, the main peak current enhancement will be strengthened dramatically.

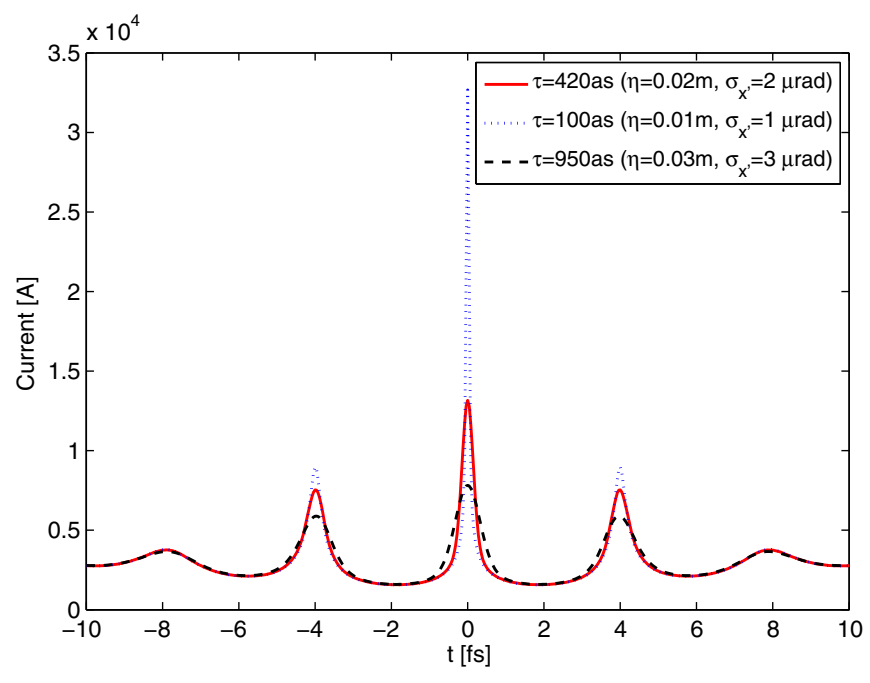

FIG. 5. Current distribution for different current width $\tau=$ 420 as (red solid line), $\tau=100$ as (blue dotted line), and $\tau=$ 950 as (black dashed line).
One thing we want to mention here is that the current width should be large enough to cover the slippage length. For current width $\tau=420 \mathrm{as}$, the main peak current is about $13 \mathrm{kA}$, and the two side peaks current is about $7.5 \mathrm{kA}$.

Furthermore, we need to choose the proper parameters for $\eta$ and $\sigma_{x^{\prime}}$ respectively. Noting that in Eq. (8), at the optimized conditions, the transverse dispersion of the dogleg $\eta$ is corresponding to the angular dispersion of the $\mathrm{AD}$ section $b_{1}$. And together with the initial beam divergence $\sigma_{x^{\prime}}$, these two parameters will have a great impact on the emittance growth in the AD section, hence will influence the gain-lengths of the main current peak and side current peaks. On the one hand, we want the gainlengths to be as short as possible to reduce the undulator length. On the other hand, we need the gain-length of the main current peak to be shorter than that of the side peaks, so that the main current peak will come to saturation earlier than the side peaks do and the final FEL radiation pulse will have a better signal-to-noise ratio.

Considering these aspects, we scan the current width $\tau$, the gain-length $L g_{0}$ of the main current peak and the gainlength ratio of side peaks to main current peak $L g_{1} / L g_{0}$ in terms of different $\eta$ and $\sigma_{x^{\prime}}$, showing the results in Fig. 6. From Fig. 6(a) one can find that the gain length of the main current peak will increase either $\eta$ is too small or $\sigma_{x^{\prime}}$ is too large, cause both of them will lead to a great emittance growth of the electron beam. And even though the main peak current will get a significant enhancement with a small product of $\eta \sigma_{x^{\prime}}$, the increase of the emittance will yet result in the increase of the gain-length, as shown in the bottom left corner of Fig. 6(a). However, the gain-length ratio of side peaks to main current peak keeps increasing when $\eta \sigma_{x^{\prime}}$ decreases, as shown in Fig. 6(b), for the main peak current will be strengthened dramatically while the side peaks are not, and they suffer the same emittance growth.

Given the analysis above, we should pick up a parametric point where $L g_{0}$ is relatively small in Fig. 6(a) and $L g_{1} / L g_{0}$ 


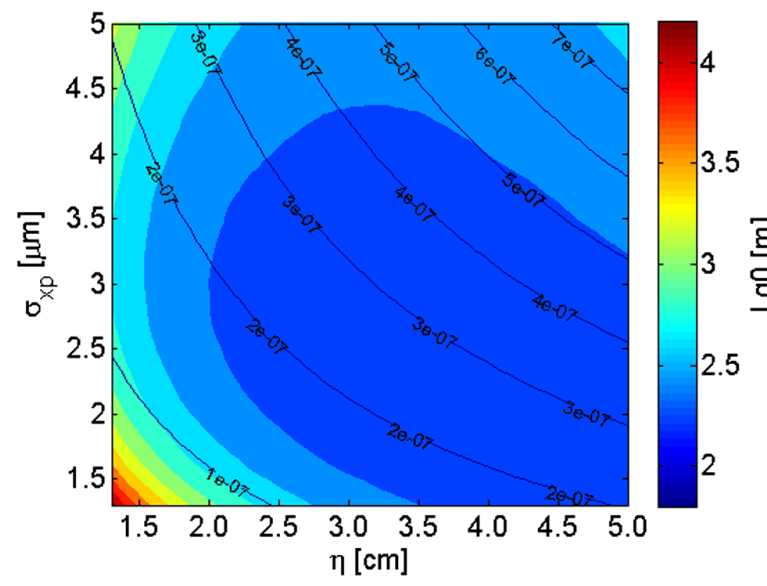

(a)

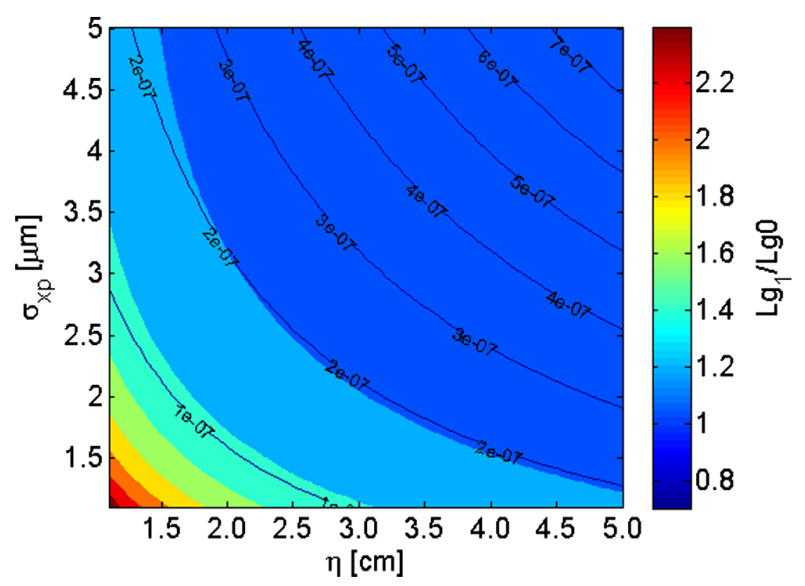

(b)

FIG. 6. (a) Gain-length $L g_{0}$ of the main current peak and (b) gain-length ratio of side peaks to main current peak $L g_{1} / L g_{0}$ in terms of different $\eta$ and $\sigma_{x^{\prime}}$. The contour lines in both figures correspond to the current width $\tau$, units in meters.

is relatively large in Fig. 6(b). As to the current width $\tau$, it should be not less than $0.1 \mu \mathrm{m}$ in our estimation, so as to the cover the whole slippage length. Eventually the transverse dispersion of the dogleg $\eta$ and the initial beam divergence $\sigma_{x^{\prime}}$ are chosen to be $0.02 \mathrm{~m}$ and $2 \mu \mathrm{rad}$ respectively. So the angular dispersion $b_{1}$ of the $\mathrm{AD}$ section is $55 \mathrm{mrad}$. And the emittance grows to be about $1.3 \mu \mathrm{m}$ after the $\mathrm{AD}$ section. The gain-length of the main current peak is about $2.4 \mathrm{~m}$. That of the side peaks is about $3.3 \mathrm{~m}$. The width of the main current peak is $1.26 \times 10^{-7} \mathrm{~m}$, which is about 420 as.

\section{SIMULATION RESULTS}

Using the parameters given above, we conduct a threedimensional simulation of the FEL gain processes with GENESIS [25]. It is worth noting that the modulation processes of the electron beam before entering the radiator section are done with a three-dimensional algorithm dealing with the dynamics of electrons [26]. At the entrance of the radiator section, the current profile and the slice energy spread $\sigma_{\gamma}$ within the central part of the electron beam are shown in Fig. 7. The results of the current profile from simulation are in agreement with the theoretical predictions depicted in the red solid line in Fig. 5, i.e., the main peak current is about $13 \mathrm{kA}$, the two side peaks current is about $7.5 \mathrm{kA}$ and the current width is about 420 as. This electron beam will then go through the undulator which resonates at $0.15 \mathrm{~nm}$. The radiation power at a distance of about $40 \mathrm{~m}$ is shown as the red solid line in Fig. 8(a). We can see the main peak power is about $25 \mathrm{GW}$. The main peak pulse duration is about 210 as (FWHM). The whole FEL pulse energy is about $5.5 \mu \mathrm{J}$, and the main peak pulse contributes to about $70 \%$ of the total.

For comparison purpose, we also conduct a FEL simulation for the normal ESASE technique. The radiation saturates at about $35 \mathrm{~m}$. The FEL pulse is shown as the blue dashed line in Fig. 8(a). The whole pulse energy is about $6 \mu \mathrm{J}$, the peak power is $30 \mathrm{GW}$, the pulse length is also about 210 as (FWHM), and the main peak pulse contributes to about $50 \%$ of the total. Figure 8 (b) illustrates the current profile and slice energy spread at the entrance of the radiator section for the normal ESASE technique. One can clearly find that for the formation of this kind of current profile, the required energy modulation strength is pretty high. Actually the peak power of the modulation laser we used here is $26 \mathrm{GW}$, which is an order of magnitude higher than that being used in our proposed scheme. The comparison results here have proved that our proposed scheme can significantly reduce the required seed laser power, and the signal-to-noise ratio in our proposed scheme is also slightly better than that in the conventional ESASE scheme, even though the saturation length will increase and the peak power will decline a little bit due to the emittance growth in our proposed scheme.

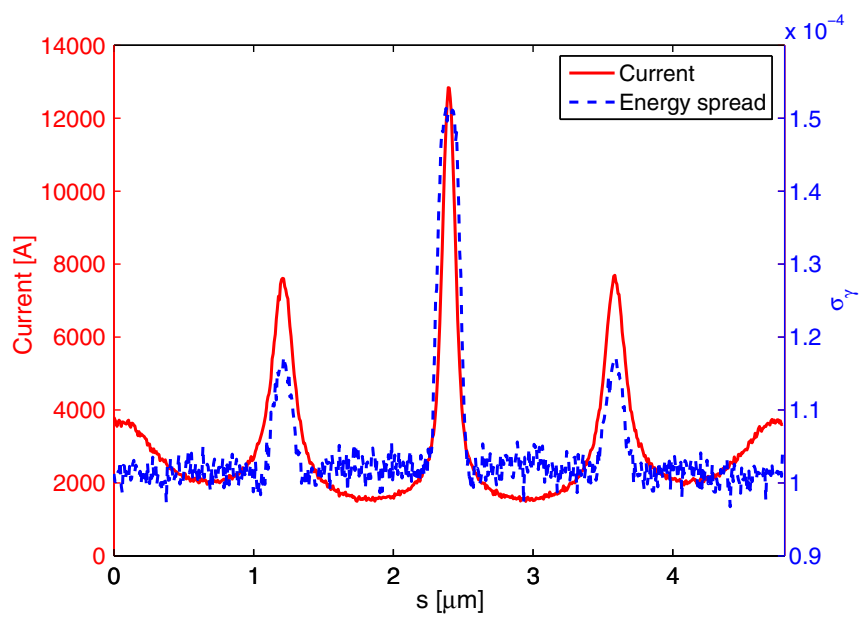

FIG. 7. Current profile (red solid line) and slice energy spread (blue dashed line) at the entrance of the undulator. 


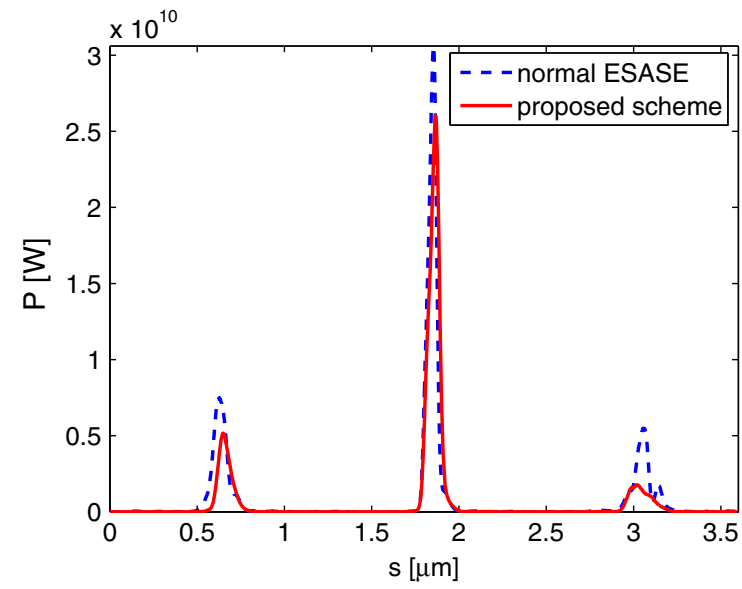

(a)

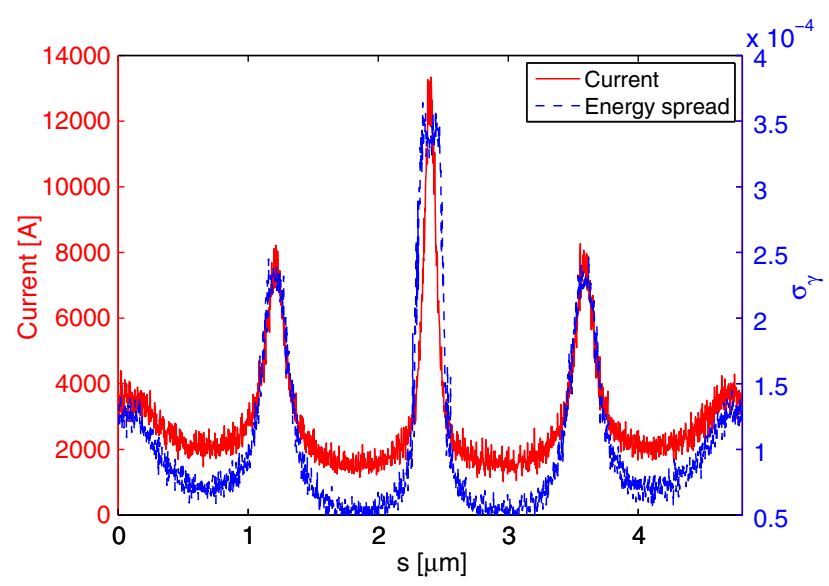

(b)

FIG. 8. (a) Radiation power for our proposed scheme at $40 \mathrm{~m}$ (red solid line) and for the normal ESASE technique at $35 \mathrm{~m}$ (blue dashed line). (b) Current profile (red solid line) and slice energy spread (blue dashed line) at the entrance of the radiator section for the normal ESASE technique.

Since the required seed laser power in our proposed scheme is relatively low, it is possible to adopt a longer wavelength laser with fewer optical cycles to further improve the signal-to-noise ratio of the final FEL pulses. For the fewer the optical cycles is, the lower the adjacent side current peaks will be, and eventually the better the signal-to-noise ratio we will get. Here we try to employ a laser pulse with the central wavelength being $2000 \mathrm{~nm}$ and the pulse length being still 4.5 fs. Therefore the FWHM of the laser pulse intensity now corresponds to only 1.2 laser cycles. The peak power of the laser is set to be $2 \mathrm{GW}$, the maximum energy modulation strength is 1.5 . The corresponding dispersion $\xi_{d}$ is $2.1 \mathrm{~mm}$. The optimized transverse dispersion of the dogleg $\eta$ and the initial beam divergence $\sigma_{x^{\prime}}$ are now chosen to be $0.025 \mathrm{~m}$ and $2.5 \mu \mathrm{rad}$ respectively. And then the angular dispersion $b_{1}$ is needed to be $84 \mathrm{mrad}$. The beam emittance after the AD section is about $1.7 \mu \mathrm{m}$ now. The gain-length of the main current peak is now $3.2 \mathrm{~m}$, that of the side peaks is $5.6 \mathrm{~m}$. And the width of the main current peak is now 650 as. The current profile and the slice energy spread at the entrance of the radiator section are shown in Fig. 9(a). In comparison with the results depicted in Fig. 7, we can see that the main peak current is still $13 \mathrm{kA}$, while the energy modulation strength of the side peaks is suppressed and the side peaks currents are now relative lower, which is only $5 \mathrm{kA}$ here. The FEL radiation pulse at $46.6 \mathrm{~m}$ is shown in Fig. 9(b). The peak power is about $35 \mathrm{GW}$, the pulse duration now is 270 as (FWHM). The whole pulse is about $10 \mu \mathrm{J}$, and the main peak pulse now holds a portion of nearly $92 \%$. We can see clearly that the signal-to-noise ratio of the final FEL pulse is now significantly improved.

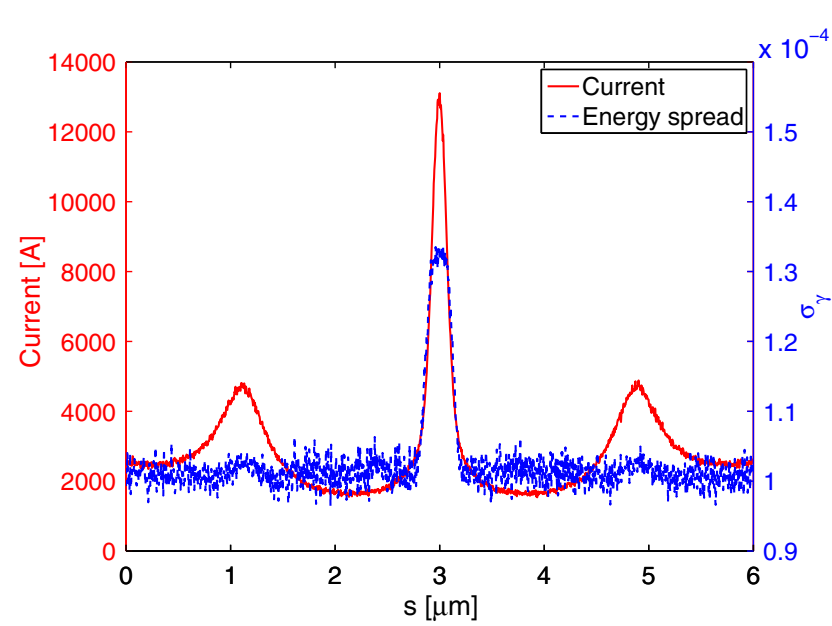

(a)

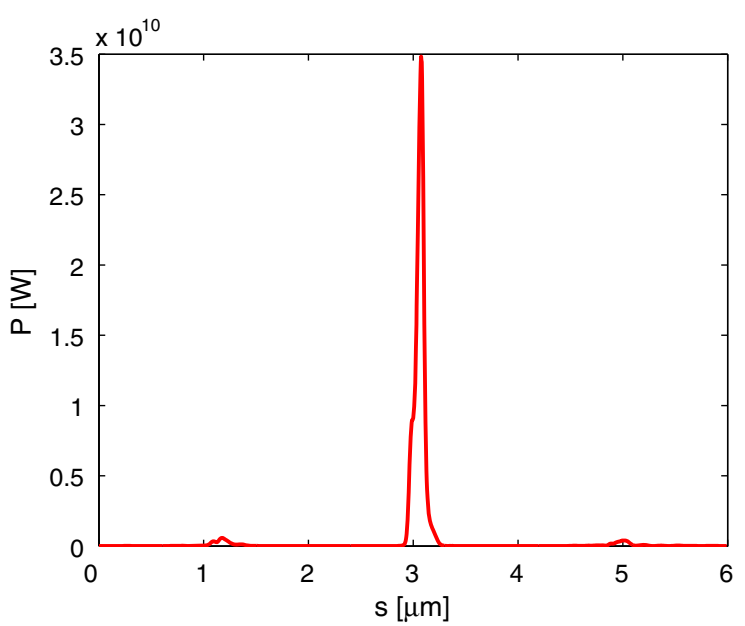

(b)

FIG. 9. (a) Current profile (red solid line) and slice energy spread (blue dashed line) at the entrance of the radiator section for the 1.2 cycles modulating laser employed in our proposed scheme. (b) Radiation power at $46.6 \mathrm{~m}$. 


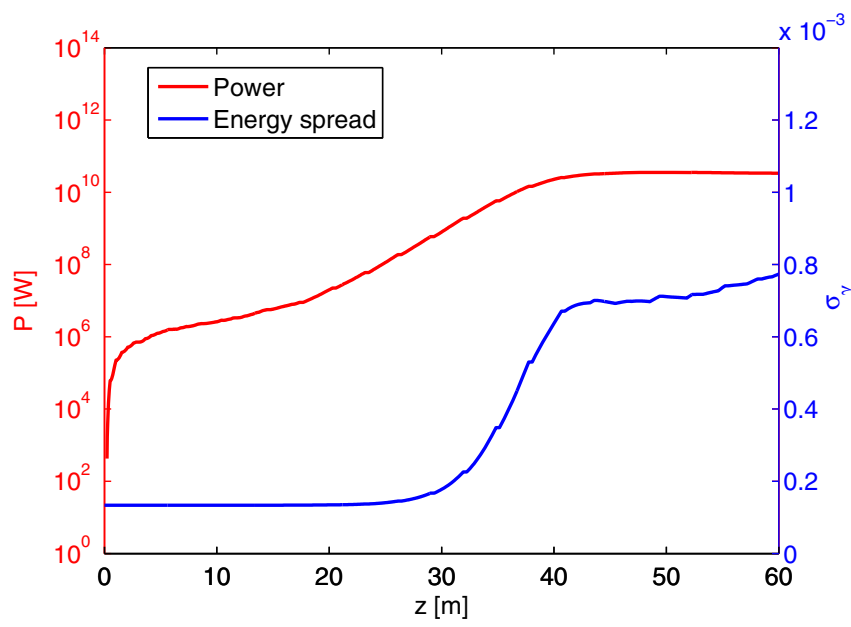

FIG. 10. The gain cure in logarithmic coordinate (red line) and the slice energy spread (blue line) of the main current peak in different distances along the undulator.

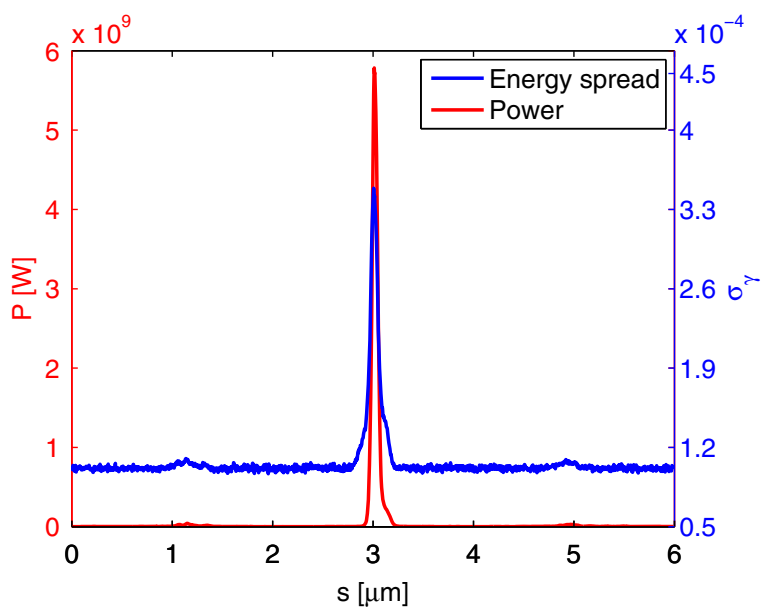

(a)

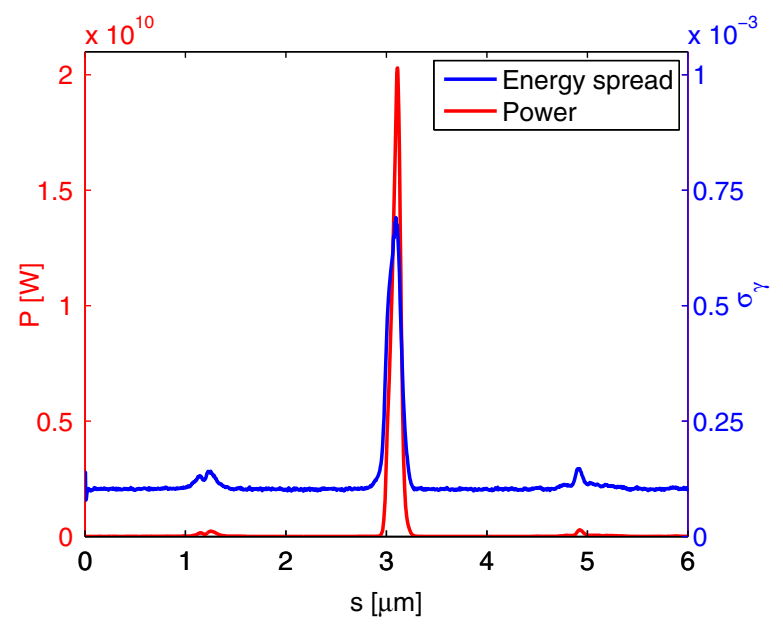

(c)
We also depict the power gain curve (red line) and the slice energy spread (blue line) of the main current peak in Fig. 10. We can see it takes about $45 \mathrm{~m}$ to reach saturation, and the energy spread at saturation is about $7 \times 10^{-4}$. Then we try to split the radiator section into two separate undulator sections, so that the electron beam can radiate independently in the two undulator sections to generate two-pulse two-color x-ray FEL. To eliminate the microbunching produced in the first undulator section and to adjust the time delay between these two-color FEL pulses, we assume a small chicane laying between the two undulator sections. Except the changes we make in the radiator section, the initial electron beam properties and the lattice configurations before the radiator section remain unchanged. Therefore at the entrance of the first undulator section, the electron beam properties are still the same as Fig. 9a. The first FEL radiation pulse is at $0.15 \mathrm{~nm}$. We stop the first FEL pulse amplification in $35 \mathrm{~m}$, at which point the

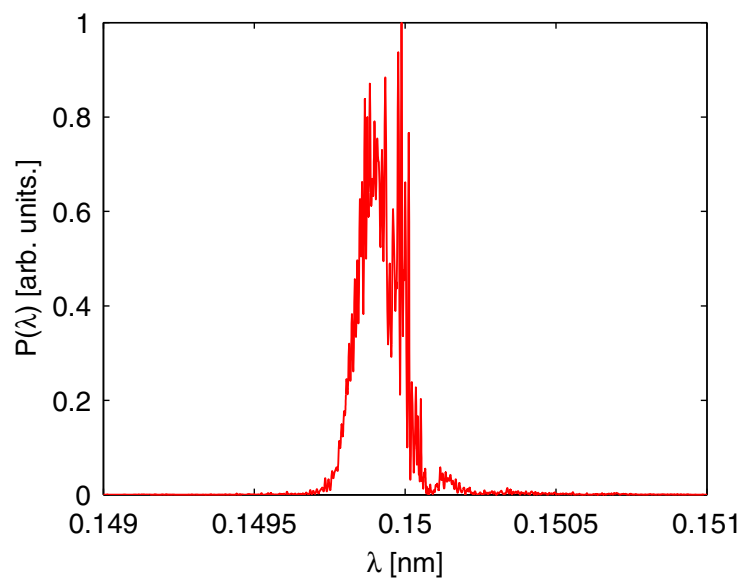

(b)

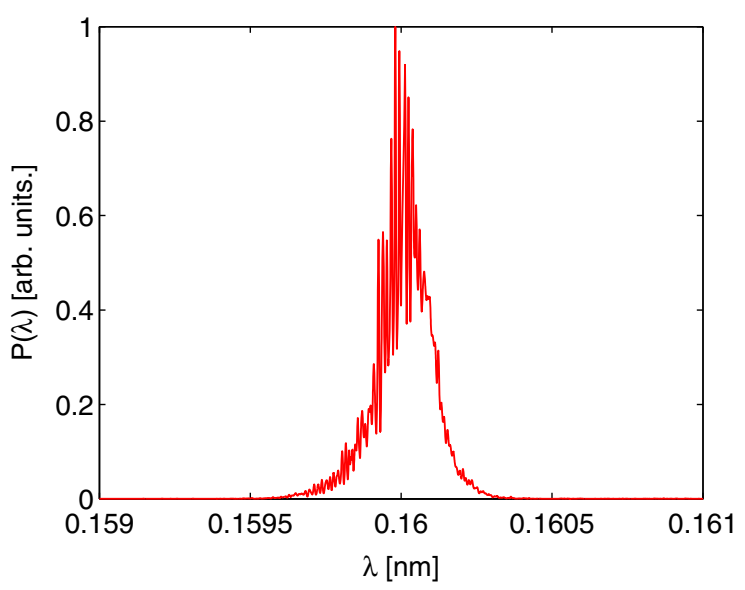

(d)

FIG. 11. (a) Power (red line) of the first FEL pulse and the energy spread of the electron beam at the end of the first undulator section. (b) Spectrum of the first FEL pulse. (c) Power (red line) of the second FEL pulse and the energy spread of the electron beam at the end of the second undulator section. (d) Spectrum of the second FEL pulse. 
energy spread of the main current peak has not increased too much. Then we send the electron beam through the small chicane, after that the second undulator section. The second FEL pulse is at $0.16 \mathrm{~nm}$. The photon energy here is about $500 \mathrm{eV}$ lower than that of the first FEL pulse. At the end of the second undulator section, we get two FEL pulses with different central wavelengths.

The power, spectrum of these two FEL pulses and the energy spread of the electron beam at the end of each undulator section are shown in Fig. 11. We can see the first FEL pulse is about $6 \mathrm{GW}$, and the energy spread of the electron beam now grows to be $3.5 \times 10^{-4}$. The signal-tonoise ratio of the first FEL pulse is about $90 \%$. The spectrum bandwidth of the first FEL pulse is $0.14 \%$. The power of the second FEL pulse is about $20 \mathrm{GW}$ in $40 \mathrm{~m}$. The energy spread of the electron beam at the end of the second undulator is about $7 \times 10^{-4}$. The signal-to-noise ratio of the second FEL pulse is about $94 \%$. The spectrum bandwidth of the second FEL pulse is about $0.11 \%$. It is worth noting that the wavelengths of these two FEL pulses are tunable by changing the gaps of the undulators. And the time delays between these two pulses are also adjustable with the help of the small chicane. Therefore the set up could meet the requirements in practical two-color pumpprobe experiments.

\section{DISCUSSIONS AND CONCLUSIONS}

We want give some comments and discuss some questions here. First, we want to address that our proposed scheme has the potential to generate ultrashort FEL pulses down to tens of attoseconds. The limitation of the pulse duration lies on the slippage effect the electron beam suffers while propagating forward with the FEL pulse. On account of this, the FEL radiation emitted from a single ultrashort current spike will not be fully amplified, which means the power for a tens-of-attoseconds FEL radiation is typically no more than $100 \mathrm{MW}$. However, combining this scheme with the mode-lock concept, we may achieve terawatt-tens-of-attoseconds $\mathrm{x}$-ray FEL pulse generation, especially on the condition of a lower power external seed laser in a high rep-rate FEL facility. Second, we want to point out that it is also possible to further increase the power and the contrast ratio of the two-pulse two-color FEL radiation pulses by using the harmonic lasing or the frequency doubler techniques [27-29]. We also want to mention here that the transverse position of the electron beam after passing through the $\mathrm{AD}$ section may have some jitters because of the inevitable central energy jitter of the electron beam. It will affect the FEL light source position and hence have impacts on the experiments. The offset factor $\delta_{\chi}$ as a function of the energy jitter $\delta_{e}$ reads approximately

$$
\delta_{\chi}=b_{1}\left(L_{1}+L_{2}\right) \delta_{e}
$$

For a conceivable CW-FEL facility, the central energy jitter in the linear accelerator will be very small, like $1 \times 10^{-4}$, then the transverse position jitter will be about $16 \mu \mathrm{m}$, depending on the lattice configurations of $b_{1}, L_{1}$, $L_{2}$. And the transverse electron beam size in our proposed scheme will increase to about $50 \mu \mathrm{m}$ due to the emittance growth. So that the transverse position jitter holds about $1 / 3$ of the transverse beam size.

In conclusion, we propose a scheme that can generate attosecond $\mathrm{x}$-ray pulses through an angular dispersion enhanced self-amplified spontaneous emission free electron laser. Our proposed scheme employs a modified ESASE scheme together with a few cycle laser pulse to manipulate the electron beam precisely. By introducing another dimension of manipulation, the needed laser power could be reduced by at least one order of magnitude, from tens or nearly hundreds of GW to only a few GW. This will help a lot in the operation of a practical FEL facility. Additionally, the reduction of the seed laser power will enable the use of a longer wavelength seed laser with fewer optical cycles in our proposed scheme, which will lead to a great improvement over the signal-to-noise ratio of the final FEL pulse. Moreover, the final FEL pulse duration could be maintained to be hundreds of attoseconds or even tens of attoseconds. And we can further use this scheme to generate two-pulse two-color attosecond FEL radiation with tunable central wavelengths and time delays, which could meet the requirements in a conceivable x-ray pump/ $\mathrm{x}$-ray probe experiment. In our simulation, we generate a single saturated FEL pulse at $0.15 \mathrm{~nm}$ with the power and the pulse duration to be $35 \mathrm{GW}$ and 270 as. The signal-tonoise ratio of this saturated FEL pulse is about $92 \%$. We also generate two FEL pulses with the central wavelengths of $0.15 \mathrm{~nm}$ and $0.16 \mathrm{~nm}$, and the corresponding powers are $6 \mathrm{GW}$ and $20 \mathrm{GW}$ with the signal-to-noise ratio of about $90 \%$ and $94 \%$ respectively.

\section{ACKNOWLEDGMENTS}

The authors would like to thank Zhen Wang, Si Chen, Tao Liu and Kaiqing Zhang for helpful discussions. This work is supported by the National Natural Science Foundation of China (Grant No. 11475250 and 11605277) and Youth Innovation Promotion Association CAS (Grant No. 2015209).

[1] A. Picn et al., Hetero-site-specific X-ray pump-probe spectroscopy for femtosecond intramolecular dynamics, Nat. Commun. 7, 11652 (2016).

[2] E. Ferrari et al., Widely tunable two-colour seeded freeelectron laser source for resonant-pump resonant-probe magnetic scattering, Nat. Commun. 7, 10343 (2016).

[3] H. Mashiko, T. Yamaguchi, K. Oguri, A. Suda, and H. Gotoh, Characterizing inner-shell with spectral phase interferometry for direct electric-field reconstruction, Nat. Commun. 5, 5599 (2014). 
[4] E. Allaria et al., Two-colour pump-probe experiments with a twin-pulse-seed extreme ultraviolet free-electron laser, Nat. Commun. 4, 2476 (2013).

[5] P. Emma et al., First lasing and operation of an ångstromwavelength free-electron laser, Nat. Photonics 4, 641 (2010).

[6] T. Ishikawa et al., A compact X-ray free-electron laser emitting in the sub-ångström region, Nat. Photonics 6, 540 (2012).

[7] E. Allaria et al., Two-stage seeded soft-X-ray free-electron laser, Nat. Photonics 7, 913 (2013).

[8] C. Pellegrini, A. Marinelli, and S. Reiche, The physics of X-ray free-electron lasers, Rev. Mod. Phys. 88, 015006 (2016).

[9] C. Bostedt, S. Boutet, D. M. Fritz, Z. Huang, H. J. Lee, H. T. Lemke, A. Robert, W. F. Schlotter, J. J. Turner, and G. J. Williams, Linac coherent light source: The first five years, Rev. Mod. Phys. 88, 015007 (2016).

[10] C. Feng and H.-X. Deng, Review of fully coherent freeelectron lasers, Nuclear Science and Techniques 29, 160 (2018).

[11] A. M. Kondratenko and E. L. Saldin, Generation of coherent radiation by a relativistic-electron beam in an undulator, Part. Accel., 10, 207 (1980).

[12] R. Bonifacio, C. Pellegrini, and L. Narducci, Collective instabilities and high-gain regime in a free electron laser, Opt. Commun. 50, 373 (1984).

[13] A. A. Zholents and W. M. Fawley, Proposal for Intense Attosecond Radiation from an X-Ray Free-Electron Laser, Phys. Rev. Lett. 92, 224801 (2004).

[14] A. A. Zholents, Method of an enhanced self-amplified spontaneous emission for x-ray free electron lasers, Phys. Rev. ST Accel. Beams 8, 040701 (2005).

[15] A. A. Zholents and G. Penn, Obtaining attosecond x-ray pulses using a self-amplified spontaneous emission free electron laser, Phys. Rev. ST Accel. Beams 8, 050704 (2005).

[16] Y. Ding, Z. Huang, D. Ratner, P. Bucksbaum, and H. Merdji, Generation of attosecond x-ray pulses with a multicycle two-color enhanced self-amplified spontaneous emission scheme, Phys. Rev. ST Accel. Beams 12, 060703 (2009).

[17] P. Emma, K. Bane, M. Cornacchia, Z. Huang, H. Schlarb, G. Stupakov, and D. Walz, Femtosecond and Subfemtosecond X-Ray Pulses from a Self-Amplified SpontaneousEmission-Based Free-Electron Laser, Phys. Rev. Lett. 92, 074801 (2004).
[18] D. Xiang, Z. Huang, and G. Stupakov, Generation of intense attosecond $\mathrm{X}$-ray pulses using ultraviolet laser induced microbunching in electron beams, Phys. Rev. ST Accel. Beams 12, 060701 (2009).

[19] S. Huang, Y. Ding, Y. Feng, E. Hemsing, Z. Huang, J. Krzywinski, A. A. Lutman, A. Marinelli, T. J. Maxwell, and D. Zhu, Generating Single-Spike Hard X-Ray Pulses with Nonlinear Bunch Compression in Free-Electron Lasers, Phys. Rev. Lett. 119, 154801 (2017).

[20] T. Tanaka, Proposal for a Pulse-Compression Scheme in X-Ray Free-Electron Lasers to Generate a Multiterawatt, Attosecond X-Ray Pulse, Phys. Rev. Lett. 110, 084801 (2013).

[21] E. Prat and S. Reiche, Simple Method to Generate Terawatt-Attosecond X-Ray Free-Electron-Laser Pulses, Phys. Rev. Lett. 114, 244801 (2015).

[22] Z. Wang, C. Feng, and Z. Zhao, Generating isolated terawatt-attosecond X-ray pulses via a chirped-laserenhanced high-gain free-electron laser, Phys. Rev. Accel. Beams 20, 040701 (2017).

[23] C. Feng and Z. Zhao, A storage ring based free-electron laser for generating ultrashort coherent EUV and X-ray radiation, Sci. Rep. 7, 4724 (2017).

[24] Z. Zhao et al., in Proceedings of the 32nd Free Electron Laser Conference, Malmö, Sweden (Max-lab, Sweden, 2010), p. 626.

[25] S. Reiche, GENESIS 1.3: a fully 3D time-dependent FEL simulation code, Nucl. Instrum. Methods Phys. Res., Sect. A 429, 243 (1999).

[26] H.-X. Deng, T.-Y. Lin, J. Yan, D. Wang, and Z.-M. Dai, Three-dimensional numerical investigations of the laserbeam interactions in an undulator, Chin. Phys. C 35, 308 (2011).

[27] E. A. Schneidmiller and M. V. Yurkov, Harmonic lasing in X-ray free electron lasers, Phys. Rev. ST Accel. Beams 15, 080702 (2012).

[28] E. A. Schneidmiller, B. Faatz, M. Kuhlmann, J. RönschSchulenburg, S. Schreiber, M. Tischer, and M. V. Yurkov, First operation of a harmonic lasing self-seeded free electron laser, Phys. Rev. Accel. Beams 20, 020705 (2017).

[29] J. Feldhaus, M. Krfer, T. Mller, J. Pflger, E. Saldin, E. Schneidmiller, and M. Yurkov, Efficient frequency doubler for the soft X-ray SASE FEL at the TESLA Test Facility, Nucl. Instrum. Methods Phys. Res., Sect. A 528, 471 (2004). 